\title{
Cancer Risk Behaviors, Cancer Beliefs and Health Information Seeking Among Under-Represented Populations in San Francisco: Differences by Sexual Orientation and Gender Identity
}

\author{
Eduardo J. Santiago-Rodríguez \\ University of California, San Francisco \\ Natalie A. Rivadeneira \\ University of California, San Francisco \\ Michelle A. DeVost \\ University of California, San Francisco \\ Urmimala Sarkar \\ University of California, San Francisco \\ Robert A. Hiatt ( $\nabla$ rahiatt145@icloud.com ) \\ University of California, San Francisco
}

\section{Research Article}

Keywords: sexual orientation and gender identity, sexual and gender minorities, health information seeking, cancer risk behaviors, cancer beliefs, San Francisco

Posted Date: August 13th, 2021

DOI: https://doi.org/10.21203/rs.3.rs-745282/v1

License: (c) (i) This work is licensed under a Creative Commons Attribution 4.0 International License.

Read Full License 


\section{Abstract \\ Background}

Compared to the general population, sexual and gender minorities (SGM) are at increased risk of some types of cancer, in part due to engagement in high-risk behaviors and lack of adequate preventive care. Understanding how SGM perceive cancer risk and their practices and preferences for accessing health information is key for improving the preventive and healthcare services they receive.

\section{Methods}

In this cross-sectional study we analyzed data from the San Francisco Health Information National Trends Survey. SGM were identified by self-report. Differences in cancer risk factors and cancer beliefs as well as health information seeking behaviors and preferences were evaluated by sexual orientation and gender identity (SOGI) using multivariable logistic regression models.

\section{Results}

Out of 1027 participants, 130 (13\%) reported being SGM. In multivariable models adjusting for age, sex at birth, education and race/ethnicity, current smoking was more common in SGM than non-SGM respondents $(\mathrm{OR}=1.62,95 \% \mathrm{Cl}=1.03,2.54)$. No differences in health information seeking behaviors, preferences and cancer beliefs were observed by SGM status. However, SGM reported significantly higher odds of feeling frustrated during the most recent health information search $(\mathrm{OR}=1.71,95 \% \mathrm{Cl}=1.14$, 2.56).

\section{Conclusions}

In this diverse urban population, SGM were more likely to be current smokers. No differences in preferences for accessing and exchanging health information were reported by SGM status. The drivers of increased feelings of frustration while seeking health information and its implications for SGM health and care merit further evaluation. Also, tobacco cessation interventions should be promoted in this community.

\section{Background}

In the U.S., it is estimated that $4.5 \%$ of the population (or 11.3 million individuals) are sexual and gender minorities (SGM) [1]. Yet, sexual orientation and gender identity (SOGI) information is not routinely collected in health surveillance systems, national surveys, medical records, and biomedical research [24]. In recent years, leading health-related organizations in the country recognized the urgency of eliminating this gap in knowledge, and designated SGM as a priority group [5-8]. In 2010, for the first 
time, the U.S. Department of Health and Human Services included SGM among the topics and objectives of the Healthy People initiative for 2020 [9]. The Institute of Medicine recommended in 2011 that "the National Institutes of Health $(\mathrm{NIH})$ should implement a research agenda designed to advance the knowledge and understanding of SGM health" [6]. In 2015, NIH established the Sexual \& Gender Minority Research Office which leads a series of initiatives to promote and support SGM research [10]. More recently, state and national surveys began including questions about SOGI [11]. Also, health care systems have been incorporating SOGI in patients' records; and population-based data sources, like the ones collected by the Census Bureau and cancer registries, have initiated work to include this information [4, $12,13]$.

Although population-level data about health outcomes by SOGI in the U.S. is limited, reports indicate that SGM have higher incidence and mortality of many diseases than the general population, including cancer $[14,15]$. SGM are at increased risk of some types of cancer, in part due to engagement in high-risk behaviors (e.g., use of tobacco products, and alcohol) and lack of adequate care across the cancer continuum [16-22]. For SGM, barriers to receiving optimal care include discrimination, stigma at individual, interpersonal, and structural levels, lack of knowledge about cancer risk factors and screening guidelines, inaccurate cancer beliefs, poor clinician-patient communication, and providers' limited knowledge of SGM health and their specific needs [23-26]. Also, the absence of information about SGM in data sources and health research in general contribute to this problem and impedes gathering evidence that allows for the development of public health interventions that improve SGM health.

According to recent estimates, $6.7 \%$ of people in the San Francisco Bay Area identify as SGM, the highest proportion of any large metropolitan area in the U.S. [27]. Conducting research in this setting represents a great opportunity for obtaining information on this vulnerable population. In this study, we assessed the relationship between SGM status and cancer risk behaviors, cancer beliefs, and health information seeking behaviors, preferences and perceptions in a multiethnic urban population in San Francisco. Understanding how SGM perceive cancer risk, and their practices and preferences for accessing health information is key for improving the healthcare services they receive and reduce health inequities in this group.

\section{Methods}

\section{Data source, study design and study population}

In this cross-sectional study we analyzed data from the San Francisco Health Information National Trends Survey (SF-HINTS). SF-HINTS was conducted in 2017 as part of the research activities of the San Francisco Cancer Initiative, a collaborative effort between health care providers, academic centers, government, community groups, and residents to reduce the burden of cancer in the city [28].

Unlike HINTS, which employs a two-stage sampling design to recruit a representative sample of the U.S., SF-HINTS used community-based snowball sampling to reach members of diverse populations often 
excluded from research [29]. Predefined proportions of the total sample corresponded to two characteristics of interest: language and race/ethnicity. Fifty-percent of all interviews were conducted in English (among those, $50 \%$ of respondents were African American) and the remainder in Cantonese or Mandarin (25\%) and Spanish (25\%). Participants of the study were reached at popular community locations in San Francisco (e.g., street markets, parks, community events) during May-September, 2017. The research team obtained informed consent and administered surveys face-to-face using iPads. Whenever participants were comfortable, they were able to take the survey on their own under the supervision of the survey administrator. Data were collected using the Research Electronic Data Capture (REDCap) web-based application hosted at the University of California, San Francisco [30, 31].

\section{Measures}

Participants were considered SGM based on self-report to the following questions: "How do you describe your sexual orientation or sexual identity?" and "What is your current gender identity?". A person was classified as SGM if for sexual orientation chose "Gay/Lesbian/Same-Gender Loving", "Bisexual" or any response other than "Straight/Heterosexual", and/or for gender identity selected "Female-toMale/Transgender Male/Trans Man," "Male-to-Female/Transgender Female/Trans Woman”, "Genderqueer" or any response other than "Male" and "Female". These questions were taken from the repository of validated questions featured in HINTS 5, Cycle 1 and the 2016 Behavioral Risk Factor Surveillance System survey [32, 33].

The outcome variables in this study were participants' health information seeking behaviors, preferences and perceptions, cancer risk behaviors and beliefs. For health information seeking behaviors participants were asked whether they have ever looked for information about health or medical topics from any source, their preferred source of information, their preferences for getting information from providers, sources used for getting health information from a provider in the last year and their perceptions during their most recent health information search.

To assess cancer risk behaviors, respondents were asked about lifetime smoking of at least 100 cigarettes (yes/no), current smoking (yes/no), use of e-cigarettes or other electronic "smoking" devices (yes/no), alcohol use (dichotomized for analysis as either everyday/weekly/monthly use of any beverage containing alcohol or non-use/once-a-year use) and physical inactivity ("any physical activities or exercises such as running, golf, gardening, or walking for exercise" yes/no).

To assess cancer beliefs, a Likert-type scale was used to measure participants' level of agreement (strongly agree, somewhat agree, somewhat disagree and strongly disagree) with the following five statements: "Everything causes cancer"; "There is not much you can do to lower your chances of getting cancer"; "There are so many recommendations about preventing cancer that it is hard to know which ones to follow"; "Cancer is most often caused by behavior or lifestyle"; and "When I think about cancer, I think about death". For analysis, responses were classified into two categories (agree, disagree). 
Additional sociodemographic and health care access variables were collected and considered in the analysis. These included age (continuous), sex at birth (male or female), race/ethnicity (Non-Hispanic White, Non-Hispanic Black, Hispanic, non-Hispanic Asian/Pacific Islander and Other), marital status (dichotomized as single/divorced/separated/widowed or married/domestic partnership/living as married), education (less than high school, completed high school and more than high school), employment status (employed, unemployed, retired and disabled), household income ( $<\$ 10 \mathrm{k}, \$ 10 \mathrm{k}$ to $<\$ 20 k, \$ 20 k$ to $<\$ 50 k$ and $\geq \$ 50 k$ ) and place of birth (U.S. or foreign-born). For access to care, variables measured were health insurance coverage (Medicaid, Medicare, private, other, or none), having usual place of care (yes/no), and times received care in the last year (0,1-2, 3-4 and more than 4).

\section{Statistical analysis}

Descriptive analyses were conducted to summarize and compare the characteristics of the population under study by SGM status. Differences in sociodemographic characteristics and health care access were evaluated using Student's t-test, Pearson's chi-squared test or Fisher's exact test. Unadjusted and adjusted logistic regression models were fitted to assess the association between SGM status and the outcomes of interest. Multivariable models included age, sex at birth, race/ethnicity and education. Regression results are reported as odds ratios (OR) with $95 \%$ confidence intervals $(95 \% \mathrm{Cl})$. For questions in which participants answered "do not know" or refused to answer, the information was treated as missing data and excluded from the analysis. All analyses were conducted in Stata (Version 15.1, College Station, TX, USA).

\section{Results}

In SF-HINTS, a total of 1027 participants were surveyed, and among them $130(13 \%)$ were classified as SGM (lesbian/gay, $n=64$; bisexual, $n=39$; transgender, $n=14$; queer/other, $n=13$ ). Compared to nonSGM, SGM were significantly younger and more educated. Also, a significantly higher proportion of SGM reported being assigned male sex at birth, Hispanic, unemployed, and not having a partner (Table 1). 
Table 1

Characteristics of participants in the San Francisco Health Information National Trends Survey by sexual orientation and gender identity

\begin{tabular}{|c|c|c|c|c|}
\hline & $\begin{array}{l}\text { All participants, } n \\
=1027\end{array}$ & $\begin{array}{l}\text { SGM, } n= \\
130\end{array}$ & $\begin{array}{l}\text { Non-SGM, } n= \\
897\end{array}$ & $\begin{array}{l}\mathrm{p}- \\
\text { value }\end{array}$ \\
\hline & $\mathrm{n}(\%)$ & $\mathrm{n}(\%)$ & $\mathrm{n}(\%)$ & \\
\hline \multicolumn{5}{|l|}{ Sociodemographic characteristics } \\
\hline \multicolumn{5}{|l|}{ Age } \\
\hline Mean, sd & $47.4(16.7)$ & $\begin{array}{l}40.5 \\
(13.3)\end{array}$ & $48.4(16.9)$ & ¿.001 \\
\hline \multicolumn{5}{|l|}{ Sex at birth } \\
\hline Male & $488(47.7)$ & $91(70.5)$ & $397(44.4)$ & $\dot{0} 001$ \\
\hline Female & $536(52.3)$ & $38(29.5)$ & $498(55.6)$ & \\
\hline \multicolumn{5}{|l|}{ Sexual orientation and gender identity } \\
\hline Heterosexual men and women & $897(87.3)$ & - & $897(100)$ & - \\
\hline Gay/Lesbian men and women & $64(6.2)$ & $64(49.2)$ & - & \\
\hline Bisexual men and women & $39(3.8)$ & $39(30.0)$ & - & \\
\hline Transgender men and women & $14(1.4)$ & $14(10.8)$ & - & \\
\hline Queer/Other & $13(1.3)$ & $13(10.0)$ & - & \\
\hline \multicolumn{5}{|l|}{ Race/ethnicity } \\
\hline Non-Hispanic White & $44(4.3)$ & $4(3.1)$ & $40(4.5)$ & $\hat{0.001}$ \\
\hline Non-Hispanic Black & $243(23.7)$ & $19(14.6)$ & $224(25.0)$ & \\
\hline Hispanic & $365(35.5)$ & $83(63.9)$ & $282(31.4)$ & \\
\hline Non-Hispanic Asian/Pacific-Islander & $317(30.9)$ & $13(10.0)$ & $304(33.9)$ & \\
\hline Other & $58(5.7)$ & $11(8.5)$ & $47(5.2)$ & \\
\hline \multicolumn{5}{|l|}{ Marital status } \\
\hline Single/Divorced/Separated/Widowed & $682(68.4)$ & $\begin{array}{l}104 \\
(81.9)\end{array}$ & $578(66.4)$ & $\hat{0}_{0.001}$ \\
\hline $\begin{array}{l}\text { Married/Domestic partnership/Living } \\
\text { as married }\end{array}$ & $315(31.6)$ & $23(18.1)$ & $292(33.6)$ & \\
\hline Education & & & & \\
\hline
\end{tabular}




\begin{tabular}{|c|c|c|c|c|}
\hline & $\begin{array}{l}\text { All participants, } n \\
=1027\end{array}$ & $\begin{array}{l}\text { SGM, } n= \\
130\end{array}$ & $\begin{array}{l}\text { Non-SGM, n = } \\
897\end{array}$ & $\begin{array}{l}\mathrm{p}- \\
\text { value }\end{array}$ \\
\hline Less than HS & $236(23.3)$ & $14(10.9)$ & $222(25.1)$ & $\begin{array}{l}<.001 \\
0.01\end{array}$ \\
\hline Completed HS & $305(30.2)$ & $28(21.9)$ & 277 (31.4) & \\
\hline More than HS & $470(46.5)$ & $86(67.2)$ & $384(43.5)$ & \\
\hline \multicolumn{5}{|l|}{ Employment status } \\
\hline Unemployed & $299(30.1)$ & $50(39.4)$ & $249(28.8)$ & $\begin{array}{l}<.001 \\
0.01\end{array}$ \\
\hline Employed & $435(43.9)$ & $60(47.2)$ & $375(43.4)$ & \\
\hline Retired & $137(13.8)$ & $3(2.4)$ & $134(15.5)$ & \\
\hline Disabled & $121(12.2)$ & $14(11.0)$ & $107(12.4)$ & \\
\hline \multicolumn{5}{|l|}{ Household income } \\
\hline$<\$ 10 k$ & $237(26.6)$ & $29(27.9)$ & $208(26.5)$ & 0.84 \\
\hline$\$ 10 k$ to $<\$ 20 k$ & $274(30.8)$ & $28(26.9)$ & $246(31.3)$ & \\
\hline$\$ 20 k$ to $<\$ 50 k$ & $221(24.8)$ & $27(26.0)$ & $194(24.7)$ & \\
\hline$\geq \$ 50 \mathrm{k}$ & $158(17.8)$ & $20(19.2)$ & $138(17.6)$ & \\
\hline Born in the US & $482(47.7)$ & $54(43.2)$ & $428(48.4)$ & 0.28 \\
\hline \multicolumn{5}{|l|}{ Access to health care } \\
\hline \multicolumn{5}{|c|}{ Health insurance coverage } \\
\hline None & $141(14.4)$ & $20(16.1)$ & $121(14.2)$ & 0.25 \\
\hline Medicaid & $167(17.1)$ & $19(15.3)$ & $148(17.3)$ & \\
\hline Medicare & $318(32.5)$ & $36(29.0)$ & $282(33.0)$ & \\
\hline Private & $236(24.1)$ & $27(21.8)$ & $209(24.4)$ & \\
\hline Other & $117(12.0)$ & $22(17.7)$ & $95(11.1)$ & \\
\hline Usual place of care & 848 (88.3) & $\begin{array}{l}110 \\
(88.0)\end{array}$ & 738 (88.4) & 0.90 \\
\hline \multicolumn{5}{|c|}{$\begin{array}{l}\text { Times received care in last } 12 \text { months } \\
\text { (no ER) }\end{array}$} \\
\hline 0 & 204 (19.9) & $23(17.7)$ & $181(20.2)$ & 0.72 \\
\hline $1-2$ & 356 (34.7) & $42(32.3)$ & $314(35.0)$ & \\
\hline
\end{tabular}




\begin{tabular}{|c|c|c|c|c|}
\hline & $\begin{array}{l}\text { All participants, } n \\
=1027\end{array}$ & $\begin{array}{l}\text { SGM, } n= \\
130\end{array}$ & $\begin{array}{l}\text { Non-SGM, n = } \\
897\end{array}$ & $\begin{array}{l}\text { p- } \\
\text { value }\end{array}$ \\
\hline $3-4$ & $258(25.1)$ & 35 (26.9) & $223(24.9)$ & \\
\hline$\geq 5$ & 209 (20.4) & $30(23.1)$ & $179(20.0)$ & \\
\hline
\end{tabular}

Results of the relationship between SGM status and cancer risk behaviors and beliefs are shown in Table 2. For cancer risk behaviors, $35 \%$ of the study population had history of smoking at least 100 cigarettes, $25 \%$ reported being current smokers, $9 \%$ said were using electronic devices to smoke, half of them used alcohol frequently and $23 \%$ reported not doing exercises during the last month. When evaluating these behaviors by SGM status using regression models, SGM had significantly higher odds of current smoking $(\mathrm{OR}=1.72,95 \% \mathrm{Cl}=1.15,2.56)$ and alcohol use $(\mathrm{OR}=2.24,95 \% \mathrm{Cl}=1.50,3.34)$ in unadjusted analysis. In multivariable models, results remained statistically significant for current smoking $(\mathrm{OR}=1.62,95 \% \mathrm{Cl}=1.03,2.54)$ but not for alcohol use $(\mathrm{OR}=1.46,95 \% \mathrm{Cl}=0.95,2.25)$. 
Table 2

Relationship between sexual orientation and gender identity and cancer risk behaviors and cancer beliefs in the San Francisco Health Information National Trends Survey

\begin{tabular}{|c|c|c|c|c|c|}
\hline & $\begin{array}{l}\text { All } \\
\text { participants, } \\
\mathrm{n}=1027\end{array}$ & $\begin{array}{l}\text { SGM, } \\
n= \\
130\end{array}$ & $\begin{array}{l}\text { Non- } \\
\text { SGM, } \\
n= \\
897\end{array}$ & $\begin{array}{l}\text { Unadjusted } \\
\text { a }\end{array}$ & $\begin{array}{l}\text { Adjusted } \\
\mathrm{ab}\end{array}$ \\
\hline & $\mathrm{n}(\%)$ & $n(\%)$ & n (\%) & $\begin{array}{l}\mathrm{OR}(95 \% \\
\mathrm{Cl})\end{array}$ & $\begin{array}{l}\mathrm{OR}(95 \% \\
\mathrm{Cl})\end{array}$ \\
\hline \multicolumn{6}{|l|}{ Cancer risk behaviors } \\
\hline $\begin{array}{l}\text { Lifetime smoking, at least } 100 \\
\text { cigarettes }\end{array}$ & 363 (35.4) & $\begin{array}{l}53 \\
(40.8)\end{array}$ & $\begin{array}{l}310 \\
(34.6)\end{array}$ & $\begin{array}{l}1.30(0.89 \\
1.90)\end{array}$ & $\begin{array}{l}1.05 \\
(0.68 \\
1.62)\end{array}$ \\
\hline Current smoking & $246(25.3)$ & $\begin{array}{l}44 \\
(34.9)\end{array}$ & $\begin{array}{l}202 \\
(23.8)\end{array}$ & $\begin{array}{l}1.72(1.15 \\
2.56)\end{array}$ & $\begin{array}{l}1.62 \\
(1.03 \\
2.54)\end{array}$ \\
\hline $\begin{array}{l}\text { Current use of e-cigarettes or other } \\
\text { electronic products }\end{array}$ & $81(8.6)$ & $\begin{array}{l}16 \\
(12.9)\end{array}$ & $\begin{array}{l}65 \\
(7.9)\end{array}$ & $\begin{array}{l}1.72(0.96 \\
3.08)\end{array}$ & $\begin{array}{l}1.24 \\
(0.64 \\
2.41)\end{array}$ \\
\hline Alcohol use ${ }^{c}$ & $493(51.8)$ & $\begin{array}{l}87 \\
(68.5)\end{array}$ & $\begin{array}{l}406 \\
(49.3)\end{array}$ & $\begin{array}{l}2.24(1.50, \\
3.34)\end{array}$ & $\begin{array}{l}1.46 \\
(0.95 \\
2.25)\end{array}$ \\
\hline Physical inactivity d & $226(22.7)$ & $\begin{array}{l}28 \\
(22.1)\end{array}$ & $\begin{array}{l}198 \\
(22.8)\end{array}$ & $\begin{array}{l}0.96(0.61 \\
1.50)\end{array}$ & $\begin{array}{l}0.97 \\
(0.60 \\
1.58)\end{array}$ \\
\hline \multicolumn{6}{|l|}{ Cancer beliefs } \\
\hline Everything causes cancer & $582(60.0)$ & $\begin{array}{l}74 \\
(57.8)\end{array}$ & $\begin{array}{l}508 \\
(60.3)\end{array}$ & $\begin{array}{l}0.90(0.62 \\
1.31)\end{array}$ & $\begin{array}{l}0.98 \\
(0.65 \\
1.47)\end{array}$ \\
\hline $\begin{array}{l}\text { There is not much you can do to lower } \\
\text { your chances of getting cancer }\end{array}$ & 387 (39.9) & $\begin{array}{l}43 \\
(34.1)\end{array}$ & $\begin{array}{l}344 \\
(40.8)\end{array}$ & $\begin{array}{l}0.75(0.51 \\
1.12)\end{array}$ & $\begin{array}{l}1.15 \\
(0.75 \\
1.76)\end{array}$ \\
\hline $\begin{array}{l}\text { There are so many recommendations } \\
\text { about preventing cancer, it is hard to } \\
\text { know which ones to follow }\end{array}$ & $691(70.2)$ & $\begin{array}{l}81 \\
(64.8)\end{array}$ & $\begin{array}{l}610 \\
(71.0)\end{array}$ & $\begin{array}{l}0.75(0.51 \\
1.12)\end{array}$ & $\begin{array}{l}0.93 \\
(0.61 \\
1.42)\end{array}$ \\
\hline $\begin{array}{l}\text { Cancer is most often caused by } \\
\text { behavior or lifestyle }\end{array}$ & $546(55.7)$ & $\begin{array}{l}68 \\
(54.8)\end{array}$ & $\begin{array}{l}478 \\
(55.8)\end{array}$ & $\begin{array}{l}0.96(0.66 \\
1.41)\end{array}$ & $\begin{array}{l}1.23 \\
(0.81 \\
1.87)\end{array}$ \\
\hline $\begin{array}{l}\text { When I think about cancer, I think about } \\
\text { death }\end{array}$ & $632(63.7)$ & $\begin{array}{l}79 \\
(61.7)\end{array}$ & $\begin{array}{l}553 \\
(63.9)\end{array}$ & $\begin{array}{l}0.91(0.62, \\
1.33)\end{array}$ & $\begin{array}{l}0.92 \\
(0.61 \\
1.39)\end{array}$ \\
\hline
\end{tabular}




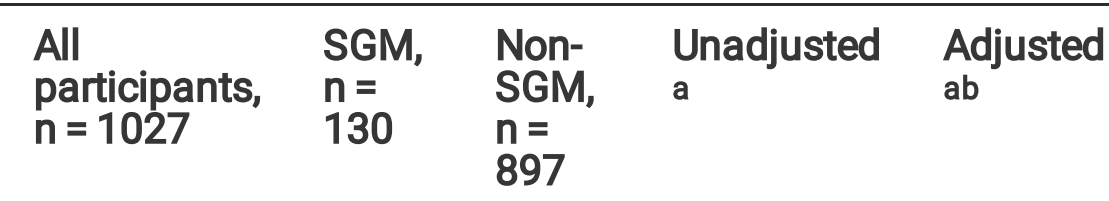

Boldface in estimates indicates statistical significance $(p<0.05)$.

${ }^{a}$ Reference group: Non-SGM

${ }^{b}$ Models adjusted for age, sex at birth, education and race/ethnicity

${ }^{\mathrm{c}}$ Everyday/weekly/monthly use of alcohol; reference group: non-use/once-a-year use

${ }^{d}$ Did not exercise during last month in activities such as running, golf, gardening, walking, not work; reference group: exercised in last month

SGM: Sexual and gender minorities

A similar pattern in the acceptance of the five cancer beliefs was observed in SGM and non-SGM. These were the percentages in descending order of popularity among all participants: "There are so many recommendations about preventing cancer that it is hard to know which ones to follow" (70\%); "When I think about cancer, I think about death" (64\%); "Everything causes cancer" (60\%); "Cancer is most often caused by behavior or lifestyle" (56\%); and "There is not much you can do to lower your chances of getting cancer" (40\%). Higher percentages of participants answering affirmatively to all these statements were observed in non-SGM, but no differences were observed by SGM status in regression models.

In Table 3 we present the results of the relationship between SGM status and health information-seeking preferences, behaviors and perceptions. Eighty-percent of study participants reported having looked for health information or medical topics in their lives, with similar frequency in SGM and non-SGM. When evaluating sources for getting health information, we found participants' most preferred choice was the internet, followed by healthcare provider, family/friend/coworker and written materials (either brochures/pamphlets or books/magazines/newspapers). The same pattern of preferences was observed in all participants irrespective of SGM status, but their use was more prevalent among SGM, particularly internet. In unadjusted analysis, the odds of reporting internet use in SGM were two times as high as in non-SGM (OR $=2.13,95 \% \mathrm{Cl}=1.47,3.09)$. In adjusted analysis SGM still had higher odds of reporting internet use, but results were not statistically significant $(\mathrm{OR}=1.40,95 \% \mathrm{Cl}=0.92,2.12)$. 
Table 3

Relationship between sexual orientation and gender identity and health information seeking in the San Francisco Health Information National Trends Survey

\begin{tabular}{|c|c|c|c|c|c|}
\hline & $\begin{array}{l}\text { All } \\
\text { participants, } \\
\mathrm{n}=1027\end{array}$ & $\begin{array}{l}\text { SGM, } \\
n= \\
130\end{array}$ & $\begin{array}{l}\text { Non- } \\
\text { SGM, n } \\
=897\end{array}$ & $\begin{array}{l}\text { Unadjusted } \\
\text { a }\end{array}$ & $\begin{array}{l}\text { Adjusted } \\
\text { ab }\end{array}$ \\
\hline & $\mathrm{n}(\%)$ & $\mathrm{n}(\%)$ & $\mathrm{n}(\%)$ & $\begin{array}{l}\text { OR }(95 \% \\
\mathrm{Cl})\end{array}$ & $\begin{array}{l}\mathrm{OR}(95 \% \\
\mathrm{Cl})\end{array}$ \\
\hline $\begin{array}{l}\text { Have ever looked for health } \\
\text { information/medical topics }\end{array}$ & $801(80.4)$ & $\begin{array}{l}111 \\
(86.1)\end{array}$ & $\begin{array}{l}690 \\
(79.6)\end{array}$ & $\begin{array}{l}1.58(0.94 \\
2.67)\end{array}$ & $\begin{array}{l}1.57 \\
(0.90 \\
2.74)\end{array}$ \\
\hline \multicolumn{6}{|l|}{$\begin{array}{l}\text { Preferred source of health } \\
\text { information }\end{array}$} \\
\hline Internet & $402(39.1)$ & $\begin{array}{l}72 \\
(55.4)\end{array}$ & $\begin{array}{l}330 \\
(36.8)\end{array}$ & $\begin{array}{l}2.13(1.47, \\
3.09)\end{array}$ & $\begin{array}{l}1.40 \\
(0.92 \\
2.12)\end{array}$ \\
\hline Healthcare provider & $369(35.9)$ & $\begin{array}{l}56 \\
(43.1)\end{array}$ & $\begin{array}{l}313 \\
(34.9)\end{array}$ & $\begin{array}{l}1.41(0.97 \\
2.05)\end{array}$ & $\begin{array}{l}1.28 \\
(0.85 \\
1.92)\end{array}$ \\
\hline Family/Friend/Coworker & $251(24.4)$ & $\begin{array}{l}37 \\
(28.5)\end{array}$ & $\begin{array}{l}214 \\
(23.9)\end{array}$ & $\begin{array}{l}1.27(0.84 \\
1.91)\end{array}$ & $\begin{array}{l}1.31 \\
(0.84 \\
2.04)\end{array}$ \\
\hline Brochure/Pamphlet & $211(20.6)$ & $\begin{array}{l}31 \\
(23.9)\end{array}$ & $\begin{array}{l}180 \\
(20.1)\end{array}$ & $\begin{array}{l}1.25(0.81 \\
1.93)\end{array}$ & $\begin{array}{l}1.45 \\
(0.91 \\
2.32)\end{array}$ \\
\hline Book/Magazine/Newspaper & $217(21.1)$ & $\begin{array}{l}23 \\
(17.7)\end{array}$ & $\begin{array}{l}194 \\
(21.6)\end{array}$ & $\begin{array}{l}0.78(0.48 \\
1.26)\end{array}$ & $\begin{array}{l}0.83 \\
(0.50 \\
1.40)\end{array}$ \\
\hline \multicolumn{6}{|l|}{$\begin{array}{l}\text { Preferences for getting health } \\
\text { information from provider }\end{array}$} \\
\hline Email & $425(41.4)$ & $\begin{array}{l}67 \\
(51.5)\end{array}$ & $\begin{array}{l}358 \\
(39.9)\end{array}$ & $\begin{array}{l}1.60(1.11, \\
2.32)\end{array}$ & $\begin{array}{l}0.96 \\
(0.63 \\
1.46)\end{array}$ \\
\hline Brochure/Pamphlet & $409(39.8)$ & $\begin{array}{l}48 \\
(36.9)\end{array}$ & $\begin{array}{l}361 \\
(40.3)\end{array}$ & $\begin{array}{l}0.87(0.59 \\
1.27)\end{array}$ & $\begin{array}{l}1.06 \\
(0.70 \\
1.61)\end{array}$ \\
\hline Text message & $271(26.4)$ & $\begin{array}{l}44 \\
(33.9)\end{array}$ & $\begin{array}{l}227 \\
(25.3)\end{array}$ & $\begin{array}{l}1.51(1.02 \\
2.24)\end{array}$ & $\begin{array}{l}1.23 \\
(0.80 \\
1.88)\end{array}$ \\
\hline Patient portal & $120(11.7)$ & $\begin{array}{l}16 \\
(12.3)\end{array}$ & $\begin{array}{l}104 \\
(11.6)\end{array}$ & $\begin{array}{l}1.07(0.61 \\
1.88)\end{array}$ & $\begin{array}{l}1.05 \\
(0.57 \\
1.92)\end{array}$ \\
\hline
\end{tabular}




\begin{tabular}{|c|c|c|c|c|c|}
\hline & $\begin{array}{l}\text { All } \\
\text { participants, } \\
\mathrm{n}=1027\end{array}$ & $\begin{array}{l}\text { SGM, } \\
n= \\
130\end{array}$ & $\begin{array}{l}\text { Non- } \\
\text { SGM, n } \\
=897\end{array}$ & $\begin{array}{l}\text { Unadjusted } \\
\text { a }\end{array}$ & $\begin{array}{l}\text { Adjusted } \\
\mathrm{ab}\end{array}$ \\
\hline DVD mailed to home & $99(9.6)$ & $\begin{array}{l}18 \\
(13.9)\end{array}$ & $81(9.0)$ & $\begin{array}{l}1.62(0.94 \\
2.80)\end{array}$ & $\begin{array}{l}1.58 \\
(0.87 \\
2.86)\end{array}$ \\
\hline \multicolumn{6}{|l|}{$\begin{array}{l}\text { Source used for getting health } \\
\text { information from provider last year }\end{array}$} \\
\hline Email & $318(31.0)$ & $\begin{array}{l}52 \\
(40.0)\end{array}$ & $\begin{array}{l}266 \\
(29.7)\end{array}$ & $\begin{array}{l}1.58(1.08, \\
2.31)\end{array}$ & $\begin{array}{l}0.93 \\
(0.60 \\
1.43)\end{array}$ \\
\hline $\begin{array}{l}\text { Text message/Instant message } \\
\text { application }\end{array}$ & $254(24.7)$ & $\begin{array}{l}39 \\
(30.0)\end{array}$ & $\begin{array}{l}215 \\
(24.0)\end{array}$ & $\begin{array}{l}1.36(0.91 \\
2.04)\end{array}$ & $\begin{array}{l}1.21 \\
(0.78 \\
1.87)\end{array}$ \\
\hline Other application on smartphone & $136(13.2)$ & $\begin{array}{l}28 \\
(21.5)\end{array}$ & $\begin{array}{l}108 \\
(12.0)\end{array}$ & $\begin{array}{l}2.01(1.26 \\
3.19)\end{array}$ & $\begin{array}{l}1.40 \\
(0.85 \\
2.31)\end{array}$ \\
\hline Video conference & $41(4.0)$ & $8(6.2)$ & $33(3.7)$ & $\begin{array}{l}1.72(0.78 \\
3.80)\end{array}$ & $\begin{array}{l}0.96 \\
(0.39 \\
2.33)\end{array}$ \\
\hline Fax & $29(2.8)$ & $6(4.6)$ & $23(2.6)$ & $\begin{array}{l}1.84(0.73 \\
4.60)\end{array}$ & $\begin{array}{l}2.02 \\
(0.73 \\
5.59)\end{array}$ \\
\hline \multicolumn{6}{|l|}{$\begin{array}{l}\text { Perceptions most recent health } \\
\text { information search }\end{array}$} \\
\hline Lot of effort getting information & $466(49.2)$ & $\begin{array}{l}60 \\
(48.8)\end{array}$ & $\begin{array}{l}406 \\
(49.2)\end{array}$ & $\begin{array}{l}0.98(0.67 \\
1.44)\end{array}$ & $\begin{array}{l}1.34 \\
(0.89 \\
2.01)\end{array}$ \\
\hline Felt frustrated getting information & $413(42.9)$ & $\begin{array}{l}62 \\
(49.2)\end{array}$ & $\begin{array}{l}351 \\
(41.9)\end{array}$ & $\begin{array}{l}1.34(0.92 \\
1.95)\end{array}$ & $\begin{array}{l}1.71 \\
(1.14, \\
2.56)\end{array}$ \\
\hline $\begin{array}{l}\text { Had concerns about quality of } \\
\text { information }\end{array}$ & $550(57.0)$ & $\begin{array}{l}81 \\
(64.3)\end{array}$ & $\begin{array}{l}469 \\
(55.9)\end{array}$ & $\begin{array}{l}1.42(0.96 \\
2.10)\end{array}$ & $\begin{array}{l}1.37 \\
(0.90 \\
2.08)\end{array}$ \\
\hline $\begin{array}{l}\text { Information was hard to } \\
\text { understand }\end{array}$ & $414(43.6)$ & $\begin{array}{l}47 \\
(39.5)\end{array}$ & $\begin{array}{l}367 \\
(44.2)\end{array}$ & $\begin{array}{l}0.83(0.56 \\
1.22)\end{array}$ & $\begin{array}{l}1.19 \\
(0.78 \\
1.81)\end{array}$ \\
\hline \multicolumn{6}{|c|}{ Boldface in estimates indicates statistical significance $(p<0.05)$. } \\
\hline \multicolumn{6}{|c|}{${ }^{b}$ Models adjusted for age, sex at birth, education and race/ethnicity } \\
\hline SGM: Sexual and gender minorities & & & & & \\
\hline
\end{tabular}


In terms of preferences for getting health information from providers, the favorite option for both SGM and non-SGM was email, followed by brochure/pamphlet, text message, patient portal and visual recorded material sent to their homes. In unadjusted models, SGM had significantly higher odds of reporting preferences for email $(\mathrm{OR}=1.60,95 \% \mathrm{Cl}=1.11,2.32)$ and text message $(\mathrm{OR}=1.51,95 \% \mathrm{Cl}=$ $1.02,2.24)$ than non-SGM, but in adjusted analyses estimates were not statistically significant $(\mathrm{OR}=0.96$, $95 \% \mathrm{Cl}=0.63,1.46 ;$ and $\mathrm{OR}=1.23,95 \% \mathrm{Cl}=0.80,1.88$, respectively).

When asked about last year use of sources for exchanging medical information with providers, the same pattern was reported by SGM and non-SGM. Email was the most used source, followed by text message/instant message applications, other applications on smartphones, video conference and fax. Although significantly higher odds of email $(\mathrm{OR}=1.58,95 \% \mathrm{Cl}=1.08,2.31)$ and non-instant messaging applications use $(\mathrm{OR}=2.01,95 \% \mathrm{Cl}=1.26,3.19)$ were reported by $\mathrm{SGM}$ in unadjusted analyses, these results were not statistically significant in multivariable models $(\mathrm{OR}=0.93,95 \% \mathrm{Cl}=0.60,1.43$, for email; and $\mathrm{OR}=1.40,95 \% \mathrm{Cl}=0.85,2.31$, for other applications). Finally, perceptions regarding the most recent search of health information was assessed and a high proportion of participants reported concerns with the following: quality of information (57\%), lot of effort getting the information (49.2\%), hard to understand information (43.6\%) and frustration while getting the information (42.9\%). When comparing these by SGM status, significantly greater odds of feeling frustration were reported by SGM in adjusted analyses $(\mathrm{OR}=1.71,95 \% \mathrm{Cl}=1.14,2.56)$.

\section{Discussion}

In this study, we evaluated the relationship between SGM status and cancer risk behaviors, cancer beliefs, and health information seeking behaviors, preferences and perceptions in a multiethnic urban population. A total of 130 participants identified themselves as SGM, allowing us to contribute to the limited but growing body of health research in this underrepresented group. Overall, we found that SGM were more likely than non-SGM to be current smokers and to report frustration while searching health information. We also found no evidence of differences between SGM and non-SGM regarding health information seeking behaviors and preferences, and cancer beliefs.

In this setting, a high prevalence of health information seeking was observed ( $\geq 80 \%)$. Also, internet was the preferred source of information, and email was the preferred method for getting information from providers. These findings confirm a trend documented in other studies, and suggest that irrespective of SGM status, participants relied on using technology for accessing and exchanging health information, over other in-person, less private and less instantaneous methods [34, 35]. These results also represent challenges and opportunities for healthcare providers and public health organizations. Having multiple sources of health information on the internet, including options not necessarily guided by science, can create confusion and misunderstandings on how SGM and the population in general use the information and deal with health-related issues [36]. On the other hand, having information that people can access whenever they need, in different media (e.g., audio, video, text), and free of cost, can be beneficial for people, especially for those belonging to the most disadvantaged social groups. Thus, in order to improve 
the quality of health information that SGM and other vulnerable populations receive on the internet, efforts should be directed to make sure they participate in the development of content and their preferences are considered in strategies for message dissemination (e.g., what websites and social media they prefer to look for health information, what organizations they trust and follow on social media).

These findings about health information seeking behaviors are particularly important in the present context of a pandemic. The COVID-19 pandemic interrupted all healthcare services, and thanks to technology patients and providers re-established communication and some clinical activities resumed $[37,38]$. Also, the internet (i.e., websites and social media) has been one of the primary sources of health information used by people, and it has helped governments and public health departments to convey their messages [39]. Therefore, having reliable voices and sources of information, with clear and culturally sensitive communication that serve the needs of vulnerable populations as SGM, is essential now and will be in the future with the likely emergence of other potential stresses on the healthcare system.

The finding that SGM engage more than non-SGM in cancer risk behaviors such as smoking, has been described previously. Using data from the 2012-2013 National Adult Tobacco Survey, Johnson et al. reported that SGM had higher prevalence of current cigarette smoking than non-SGM [16]. Also, Gonzalez et al. analyzed data from the National Health Interview Survey for years 2013 and 2014, and found that SGM reported higher prevalence of both moderate and heavy current smoking than non-SGM [21]. The presence of several factors have been proposed as possible explanations to the higher prevalence of smoking among SGM, including stress, depression, alcohol use and low socioeconomic status [40]. In our analysis, smoking remained significantly elevated even after adjustment for education as a proxy for socioeconomic status. Recent research also found that SGM are more likely than non-SGM to be exposed to tobacco-related marketing and social media, and exposure to these messages was associated with tobacco use [41]. With the high use of internet and preferences for communicating with providers observed in this study (i.e., email, text messaging apps, video conference), smoking cessation interventions that incorporate these strategies should be explored in this community.

In the evaluation of participants' perceptions during the most recent health information search, we found that SGM reported higher odds of frustration than non-SGM. To our knowledge no other studies have described this in the past. Frustration might be a barrier for SGM obtaining the information they need and follow recommendations, as these feelings tend to discourage people from further searches [42]. This finding made us consider a set of key questions that remain unanswered. What are the drivers of frustration among SGM while seeking health information? Do SGM have difficulties in accessing the information? Do SGM find the information they need? Do the information that SGM look for exist? Is the content on the material found inclusive to all? Do SGM consider the information they found suit their needs? Is it trustworthy? Qualitative research methods are better equipped to obtain answers to these questions, and should be considered as a next step for studies on this topic. 
This study has several limitations. First, misclassification of SOGI could be present in this study. Although participants had the opportunity of completing the survey on their own, and minimize the likelihood of underreported SGM status, all questionnaires were not self-administered. Misclassification of the different outcomes is also possible, especially for questions that people can feel that an answer is more acceptable than others (e.g., social desirability bias on cancer risk behaviors questions). Second, we recognize the importance of documenting differences within SGM subgroups (e.g., in this study, bisexual individuals reported higher prevalence of cancer risk behaviors and cancer fatalistic views than other subgroups; see Supplemental Table), but we consolidated all SGM in only one group to increase statistical power. Third, despite these efforts, we believe our analyses were still underpowered, as evidenced in the wide confidence intervals of some estimates. Fourth, in our interest to study a hard-toreach population, we employed a non-probabilistic sampling method, but we did not include important information throughout the process (i.e., seeds, connections) that would allow us to use a more appropriate analytic strategy for this type of sampling design (as in respondent-driven sampling) [43]. Lastly, these results may not be generalizable to other settings.

\section{Conclusions}

In this diverse urban population, a preference in the use of technology for accessing and exchanging health information was reported, irrespective of SGM status. Tobacco cessation interventions targeting SGM should be promoted in this setting, and the use of internet and instantaneous messaging applications should be considered. Also, drivers of feeling frustration while seeking health information and its implications for SGM health and care merit further evaluation. Finally, although progress has been made in collecting SOGI data and including SGM in studies, sustained efforts are needed until all datasets, and research endeavors include this information regularly.

\section{Abbreviations}

SGM

Sexual and gender minorities; SOGI:Sexual orientation and gender identity; NIH:National Institutes of Health; SF-HINTS:San Francisco Health Information National Trends Survey; REDCap:Research Electronic Data Capture; Cl:Confidence intervals; ER:Emergency room; HS:High school

\section{Declarations}

\section{Ethics approval and consent to participate}

The Internal Review Board of the University of California San Francisco approved this study (number: 1620707). Study methods were carried out in accordance with relevant ethical guidelines and regulations. All study participants provided informed consent after being briefed on study purpose and procedures.

\section{Consent for publication}


Not applicable.

\section{Availability of data and materials}

The dataset used and analyzed during the current study is available from the corresponding author on reasonable request.

\section{Competing interests}

The authors declare that they have no competing interests.

\section{Funding}

This work was supported by the National Institute on Aging under Grant T32AG049663; National Cancer Institute Population Cancer Center Support Grant P30CA082103 and NIH Midcareer Investigator Award in Patient-Oriented Research under Grant K24CA212294. The content and views in this manuscript are those of the authors and does not necessarily represent the official policy of the NIH. The NIH did not participate in any part of the study design, data collection, analysis, or interpretation, or manuscript writing.

\section{Authors' contributions}

EJS was responsible for data analysis, interpretation of results, and drafting of the manuscript. NAR collected the data, provided critical feedback and edited the manuscript. MAD provided critical feedback and edited the manuscript. US and RAH obtained funding, conceived the study, acquired the data, contributed to interpretation of results, and reviewed and edited the manuscript. All authors read and approved the final manuscript.

\section{Acknowledgements}

The authors would like to thank all the individuals and organizations that have participated in the design and implementation of the SF-HINTS, including Tung Nguyen, Rena Pasick, Anna María Nápoles, Mekhala Hoskote, Gem M. Le, Pamela Williams, Cynthia Cheung, Corina Liew, Alejo Perez-Stable Husni, Francisco Quintanilla.

\section{References}

1. Conron KJ, Goldberg SK. Adult LGBT population in the United States. The Williams Institute, UCLA, Los Angeles, CA; 2020. https://williamsinstitute.law.ucla.edu/wp-content/uploads/LGBT-Adult-USPop-Jul-2020.pdf. Accessed April 20, 2021.

2. Boehmer U, Elk R. LGBT populations and cancer: Is it an ignored epidemic? LGBT Health. 2016;3(1):1-2. https://doi.org/10.1089/lgbt.2015.0137. 
3. Bowen DJ, Boehmer U. The lack of cancer surveillance data on sexual minorities and strategies for change. Cancer Causes Control. 2007;18(4):343-9. https://doi.org/10.1007/s10552-007-0115-1.

4. Baker KE, Streed CG, Durso LE. Ensuring that LGBTQI + people count - Collecting data on sexual orientation, gender identity, and intersex status. N Engl J Med. 2021;384(13):1184-6. https://doi.org/10.1056/NEJMp2032447.

5. Wender R, Sharpe KB, Westmaas JL, Patel AV. The American Cancer Society's approach to addressing the cancer burden in the LGBT community. LGBT Health. 2016;3(1):15-8. https://doi.org/10.1089/lgbt.2015.0089.

6. Institute of Medicine. The health of lesbian, gay, bisexual, and transgender people: building a foundation for better understanding. Washington, DC: The National Academies Press; 2011. https://doi.org/10.17226/13128.

7. National Institute on Minority Health and Health Disparities. Director's message: Sexual and gender minorities formally designated as a health disparity population for research purposes. 2016. https://www.nimhd.nih.gov/about/directors-corner/messages/message_10-06-16.html. Accessed April 29, 2021.

8. Griggs J, Maingi S, Blinder V, Denduluri N, Khorana AA, Norton L, et al. American Society of Clinical Oncology position statement: Strategies for reducing cancer health disparities among sexual and gender minority populations. J Clin Oncol. 2017;35(19):2203-8. https://doi.org/10.1200/JC0.2016.72.0441.

9. US Department of Health and Human Services. HHS announces the nation's new health promotion and disease prevention agenda. 2010.

https://www.healthypeople.gov/sites/default/files/DefaultPressRelease_1.pdf. Accessed April 28, 2021.

10. National Institutes of Health. Sexual \& Gender Minority Research Office website. https://dpcpsi.nih.gov/sgmro. Accessed April 28, 2021.

11. Federal Interagency Working Group on Improving Measurement of Sexual Orientation and Gender Identity in Federal Surveys: Evaluations of sexual orientation and gender identity survey measures: What have we learned? 2016.

https://dpcpsi.nih.gov/sites/default/files/Evaluations_of_SOGI_Questions_20160923_508.pdf. Accessed April 29, 2021.

12. Gomez SL, Duffy C, Griggs JJ, John EM. Surveillance of cancer among sexual and gender minority populations: Where are we and where do we need to go? Cancer. 2019;125(24):4360-2. https://doi.org/10.1002/cncr.32384.

13. The United States Census Bureau. 2020 Census: LGBTQ+. https://www.census.gov/newsroom/press-kits/2020/2020-census-lgbtq.html. Accessed April 29, 2021.

14. Trinh M-H, Agénor M, Austin SB, Jackson CL. Health and healthcare disparities among U.S. women and men at the intersection of sexual orientation and race/ethnicity: a nationally representative 
cross-sectional study. BMC Public Health. 2017;17(1):964. https://doi.org/10.1186/s12889-0174937-9.

15. Quinn GP, Sanchez JA, Sutton SK, Vadaparampil ST, Nguyen GT, Green BL, et al. Cancer and lesbian, gay, bisexual, transgender/transsexual, and queer/questioning (LGBTQ) populations: Cancer and sexual minorities. CA Cancer J Clin. 2015;65(5):384-400. https://doi.org/10.3322/caac.21288.

16. Johnson SE, Holder-Hayes E, Tessman GK, King BA, Alexander T, Zhao X. Tobacco product use among sexual minority adults. Am J Prev Med. 2016;50(4):e91-e100. https://doi.org/10.1016/j.amepre.2015.07.041.

17. Wheldon CW, Kaufman AR, Kasza KA, Moser RP. Tobacco use among adults by sexual orientation: Findings from the Population Assessment of Tobacco and Health Study. LGBT Health. 2018;5(1):33-44. https://doi.org/10.1089/lgbt.2017.0175.

18. Obedin-Maliver J. Time to change: Supporting sexual and gender minority people-An underserved, understudied cancer risk population. J Natl Compr Canc Netw. 2017;15(11):1305-8. https://doi.org/10.6004/jnccn.2017.7050.

19. Jennings L, Barcelos C, McWilliams C, Malecki K. Inequalities in lesbian, gay, bisexual, and transgender (LGBT) health and health care access and utilization in Wisconsin. Prev Med Rep. 2019;14:100864. https://doi.org/10.1016/j.pmedr.2019.100864.

20. Cathcart-Rake EJ. Cancer in sexual and gender minority patients: Are we addressing their needs? Curr Oncol Rep. 2018;20(11):85. https://doi.org/10.1007/s11912-018-0737-3.

21. Gonzales G, Przedworski J, Henning-Smith C. Comparison of health and health risk factors between lesbian, gay, and bisexual adults and heterosexual adults in the United States: Results from the National Health Interview Survey. JAMA Intern Med. 2016;176(9):1344. https://doi.org/10.1001/jamainternmed.2016.3432.

22. Blosnich JR, Farmer GW, Lee JGL, Silenzio VMB, Bowen DJ. Health inequalities among sexual minority adults. Am J Prev Med. 2014;46(4):337-349. https://doi.org/10.1016/j.amepre.2013.11.010.

23. National Academies of Sciences, Engineering, and Medicine. Committee on Understanding the WellBeing of Sexual and Gender Diverse Populations, Committee on Population, Division of Behavioral and Social Sciences and Education. Understanding the well-being of LGBTQI + populations. Washington, DC: National Academies Press; 2020:25877. https://doi.org/10.17226/25877.

24. Hudson J, Schabath MB, Sanchez J, Sutton S, Simmons VN, Vadaparampil ST, et al. Sexual and gender minority issues across NCCN Guidelines: Results from a national survey. J Natl Compr Canc Netw. 2017;15(11):1379-82. https://doi.org/10.6004/jnccn.2017.0169.

25. Schabath MB, Blackburn CA, Sutter ME, Kanetsky PS, Vadaparampil ST, Simmons VN, et al. National survey of oncologists at National Cancer Institute-designated comprehensive cancer centers: Attitudes, knowledge, and practice behaviors about LGBTQ patients with cancer. J Clin Oncol. 2019;37(7):547-58. https://doi.org/10.1200/JC0.18.00551. 
26. Langston ME, Fuzzell L, Lewis-Thames MW, Khan S, Moore JX. Disparities in health informationseeking behaviors and fatalistic views of cancer by sexual orientation identity: A nationally representative study of adults in the United States. LGBT Health. 2019;6(4):192-201. https://doi.org/10.1089/lgbt.2018.0112.

27. Conron KJ, Luhur W, Goldberg SK. LGBT adults in large US metropolitan areas. 2021. https://escholarship.org/uc/item/84q0f822\#main. Accessed April 30, 2021.

28. Hiatt RA, Sibley A, Fejerman L, Glantz S, Nguyen T, Pasick R, et al. The San Francisco Cancer Initiative: A community effort to reduce the population burden of cancer. Health Aff (Millwood). 2018;37(1):54-61. https://doi.org/10.1377/hlthaff.2017.1260.

29. Rivadeneira NA, Hoskote M, Le GM, Nguyen TT, Napoles AM, Pasick RJ, et al. Advancing cancer control in San Francisco: Cancer screening in under-represented populations. Am J Prev Med. 2020;58(1):e1-e9. https://doi.org/10.1016/j.amepre.2019.08.024.

30. Harris PA, Taylor R, Thielke R, Payne J, Gonzalez N, Conde JG. Research electronic data capture (REDCap)-A metadata-driven methodology and workflow process for providing translational research informatics support. J Biomed Inform. 2009;42(2):377-81.

https://doi.org/10.1016/j.jbi.2008.08.010.

31. Harris PA, Taylor R, Minor BL, Elliott V, Fernandez M, O'Neal L, et al. The REDCap consortium: Building an international community of software platform partners. J Biomed Inform. 2019;95:103208. https://doi.org/10.1016/j.jbi.2019.103208.

32. National Cancer Institute. Health Information National Trends Survey 5, Cycle 1. 2017. https://hints.cancer.gov/docs/Instruments/HINTS5_Cycle1_Annotated_Instrument_English.pdf. Accessed April 29, 2021.

33. Center for Disease Control and Prevention. 2016 Behavioral Risk Factor Surveillance System Questionnaire. 2015. https://www.cdc.gov/brfss/questionnaires/pdfques/2016_BRFSS_Questionnaire_FINAL.pdf. Accessed April 29, 2021.

34. Hesse BW, Nelson DE, Kreps GL, Croyle RT, Arora NK, Rimer BK, et al. Trust and sources of health information. The impact of the internet and its implications for health care providers: Findings from the first Health Information National Trends Survey. Arch Intern Med. 2005;165(22):2618. https://doi.org/10.1001/archinte.165.22.2618.

35. Jabson JM, Patterson JG, Kamen C. Understanding health information seeking on the internet among sexual minority people: Cross-sectional analysis from the Health Information National Trends Survey. JMIR Public Health Surveill. 2017;3(2):e39. https://doi.org/10.2196/publichealth.7526.

36. Battineni G, Baldoni S, Chintalapudi N, Sagaro GG, Pallotta G, Nittari G, et al. Factors affecting the quality and reliability of online health information. Digit Health. 2020;6:205520762094899. https://doi.org/10.1177/2055207620948996.

37. Bashshur R, Doarn CR, Frenk JM, Kvedar JC, Woolliscroft JO. Telemedicine and the COVID-19 pandemic, lessons for the future. Telemed E-Health. 2020;26(5):571-3. https://doi.org/10.1089/tmj.2020.29040.rb. 
38. Hollander JE, Carr BG. Virtually perfect? Telemedicine for Covid-19. N Engl J Med. 2020;382(18):1679-81. https://doi.org/10.1056/NEJMp2003539.

39. Fan KS, Ghani SA, Machairas N, Lenti L, Fan KH, Richardson D, et al. COVID-19 prevention and treatment information on the internet: a systematic analysis and quality assessment. BMJ Open. 2020;10(9):e040487. https://doi.org/10.1136/bmjopen-2020-040487.

40. Blosnich J, Lee JGL, Horn K. A systematic review of the aetiology of tobacco disparities for sexual minorities. Tob Control. 2013;22(2):66-73. https://doi.org/10.1136/tobaccocontrol-2011-050181.

41. Emory K, Buchting FO, Trinidad DR, Vera L, Emery SL. Lesbian, gay, bisexual, and transgender (LGBT) view it differently than non-LGBT: Exposure to tobacco-related couponing, E-cigarette advertisements, and anti-tobacco messages on social and traditional media. Nicotine Tob Res. 2019;21(4):513-22. https://doi.org/10.1093/ntr/nty049.

42. McCloud RF, Okechukwu CA, Sorensen G, Viswanath K. Beyond access: barriers to internet health information seeking among the urban poor. J Am Med Inform Assoc. 2016;23(6):1053-9. https://doi.org/10.1093/jamia/ocv204.

43. Heckathorn DD. Respondent-driven sampling: A new approach to the study of hidden populations. Soc Prob. 1997; 44(2):174-99. https://doi.org/10.2307/3096941.

\section{Supplementary Files}

This is a list of supplementary files associated with this preprint. Click to download.

- SupplementaryMaterialBMCPublicHealth.docx 